\title{
HAUSDORFF MEASURES AND KMS STATES
}

\author{
MARIUS IONESCU AND ALEX KUMJIAN
}

\begin{abstract}
Given a compact metric space $X$ and a local homeomorphism $T: X \rightarrow X$ satisfying a local scaling property, we show that the Hausdorff measure on $X$ gives rise to a KMS state on the $C^{*}$-algebra naturally associated to the pair $(X, T)$ such that the inverse temperature coincides with the Hausdorff dimension. We prove that the KMS state is unique under some mild hypotheses. We use our results to describe KMS states on Cuntz algebras, graph algebras, and certain $C^{*}$-algebras associated to fractafolds.
\end{abstract}

\section{INTRODUCTION}

This note arises from a simple observation which relates to the computation of the Hausdorff dimension $\operatorname{dim} X$ of a self-similar space $X$ arising from an iterated function system that satsifies the open set condition and has similarity ratios $r_{1}, \ldots, r_{n} \in(0,1) ; \operatorname{dim} X$ is the unique positive number $s$ satisfying

$$
1=r_{1}^{s}+\cdots+r_{n}^{s}
$$

(see [5, Section 6.4]). But this agrees with the condition that ensures that a certain one-parameter automorphism group on the Cuntz algebra $\mathcal{O}_{n}$ has a KMS state. Let $\alpha: \mathbb{R} \rightarrow \operatorname{Aut}\left(\mathcal{O}_{n}\right)$ be defined such that $\alpha_{t}\left(S_{j}\right)=e^{i t \lambda_{j}} S_{j}$ for $j=1,2, \ldots, n$; then by [ 6 . Proposition 2.2] there is a KMS state for $\alpha$ at inverse temperature $\beta$ iff

$$
1=e^{-\beta \lambda_{1}}+\cdots+e^{-\beta \lambda_{n}} .
$$

If we set $r_{j}=e^{-\lambda_{j}}$ for $j=1, \ldots, n$ and $s=\beta$, the two conditions coincide (see Example 4.1 for more details). It is the purpose of this note to account for this coincidence and explore more examples. To accomplish this we apply groupoid methods from [16] and [13] (see also [20]).

In 1967 Haag, Hugenholtz and Winnink (see 8]) discovered the relevance of the KMS condition to the $C^{*}$-algebraic formulation of equilibrium states in quantum statistical mechanics. Thereafter the KMS states of a $C^{*}$-algebra with respect to a natural one-parameter automorphism group (regarded as time evolution) have played a key role in the development of the theory. Many of the basic facts may be found in the text by Bratteli and Robinson (see [1]).

Given a compact metric space $X$, a local homeomorphism $T: X \rightarrow X$ is said to satisfy the local scaling condition (see Definition $3.1 p$ if $(x, y) \mapsto \frac{\rho(T x, T y)}{\rho(x, y)}$ extends to a continuous function on $X \times X$ that is strictly positive on the diagonal. In this case, there is a continuous function $\varphi \in C(X, \mathbb{R})$ such that for all $x \in X$,

$$
e^{\varphi(x)}=\lim _{y \rightarrow x} \frac{\rho(T x, T y)}{\rho(x, y)} .
$$

This work was partially supported by a grant from the Simons Foundation (\#209277 to Marius Ionescu). 
Then $\varphi$ gives rise to a continuous real-valued cocycle $c_{\varphi}$ on the Renault-Deaconu groupoid $G$ which in turn defines a one-parameter action $\alpha$ on $C^{*}(G)$, the associated groupoid $C^{*}$-algebra.

Our main result (see Theorem 3.4) asserts that if $T: X \rightarrow X$ satisfies the local scaling condition, then the Hausdorff measure may be used to define an $(\alpha, \beta)$-KMS state on $C^{*}(G)$ where $\beta$ is the Hausdorff dimension of $X$. We next obtain conditions for the uniqueness of the KMS state (see Proposition 3.5. If $\varphi$ is constant, we derive a simple equation involving the topological entropy of $T$, the Hausdorff dimension of $X$ and the scaling constant (see Proposition 3.6).

The remainder of the paper is devoted to applications of our results to a number of examples. The last example, which is based on the Sierpinski octafold, is perhaps the most interesting. Although it does not satisfy the hypotheses of our main result, the conclusions hold. This suggests that it should be possible to weaken the hypotheses in our main result.

\section{Preliminaries}

Let $(X, \rho)$ be a metric space and let $s>0$. For a set $F \subset X$ and $\varepsilon>0$, a countable cover $\mathcal{A}$ of $F$ is called an $\varepsilon$-cover of $F$ iff $\operatorname{diam} A \leq \varepsilon$ for all $A \in \mathcal{A}$. Define

$$
\bar{\mu}_{\varepsilon}^{s}(F)=\inf \sum_{A \in \mathcal{A}}(\operatorname{diam} A)^{s},
$$

where the infimum is over all countable $\varepsilon$-covers $\mathcal{A}$ of the set $F$, Section 6.1$]$. Then $\bar{\mu}_{\varepsilon}^{s}$ is decreasing with respect to $\varepsilon$ and

$$
\bar{\mu}^{s}(F)=\lim _{\varepsilon \rightarrow 0} \bar{\mu}_{\varepsilon}^{s}(F)
$$

is a metric outer measure on $X$. Let $\mu^{s}$ be the Borel measure defined by $\bar{\mu}^{s}$.

Let $s, t>0$ such that $s<t$. It is well known ([5, Theorem 6.1.6]) that if $\mu^{t}(F)>0$ then $\mu^{s}(F)=\infty$ and if $\mu^{s}(F)<\infty$ then $\mu^{t}(F)=0$. The Hausdorff dimension $\operatorname{dim} F$ of a set $F$ is the unique number $s_{0} \in[0, \infty]$ such that $\mu^{s}(F)=\infty$ for all $s<s_{0}$ and $\mu^{s}(F)=0$ for all $s>s_{0}$. If $s=\operatorname{dim} X$, then we call $\mu^{s}$ the Hausdorff measure on $X$. In the following we assume that $0<s<\infty$ and $0<\mu^{s}(X)<\infty$.

We say $G$ is a groupoid ([16]) if there is a subset $G^{(2)}$ of $G \times G$, a map $(x, y) \mapsto x y$ from $G^{(2)}$ to $G$ and a involution $x \mapsto x^{-1}$ on $G$ such that the following conditions hold:

(1) If $(x, y)$ and $(y, z)$ are in $G^{(2)}$, then so are $(x y, z)$ and $(x, y z)$, and the equation $(x y) z=x(y z)$ is satisfied;

(2) For all $x \in G,\left(x^{-1}, x\right) \in G^{(2)}$ and if $(x, y) \in G^{(2)}$, then $x^{-1}(x y)=y$ and $(x y) y^{-1}=x$.

The maps $r$ and $s$ on $G$, defined by the formulae $r(x)=x x^{-1}$ and $s(x)=x^{-1} x$ are called the range and source maps. Then $G^{(0)}:=r(G)=s(G)$ is called the unit space of $G$. The groupoid $G$ is called a topological groupoid in case $G$ is a groupoid with a topology such that the multiplication and the inverse maps are continuous, where the topology on $G^{(2)}$ is the relative product topology. The groupoids that we consider in this note are Hausdorff and locally compact.

A left Haar system ([16]) on a topological groupoid $G$ is a family $\left\{\lambda^{u}\right\}_{u \in G^{(0)}}$ of non-negative Radon measures on $G$ such that

(1) $\operatorname{supp}\left(\lambda^{u}\right)=G^{u}, u \in G^{(0)}$; 
(2) for $f \in C_{c}(G)$, the function

$$
u \mapsto \int f d \lambda^{u}
$$

on $G^{(0)}$ is in $C_{c}\left(G^{(0)}\right)$; and

(3) for $x \in G, \int f(x y) d \lambda^{s(x)}(y)=\int f(y) d \lambda^{r(x)}$.

Once a Haar system $\left\{\lambda^{y}\right\}_{u \in G^{(0)}}$ has been specified on a groupoid, one can define an involutive algebraic structure on $C_{c}(G)$ by the formulae

$$
f * g(y)=\int f(x) g\left(x^{-1} y\right) d \lambda^{r(y)}
$$

and

$$
f^{*}(x)=\overline{f\left(x^{-1}\right)} .
$$

The groupoid $C^{*}$-algebra $C^{*}(G)$ is the completion of $C_{c}(G)$ under the universal norm (see [16] for details).

Assume that $T: X \rightarrow X$ is a local homeomorphism. The Renault-Deaconu groupoid associated to the pair $(X, T)$ (see [17, [4]) is defined via

$$
G:=\left\{(x, m-n, y): T^{m}(x)=T^{n}(y)\right\} \subset X \times \mathbb{Z} \times X .
$$

Two triples $\left(x_{1}, n_{1}, y_{1}\right)$ and $\left(x_{2}, n_{2}, y_{2}\right)$ are composable if and only if $y_{1}=x_{2}$, and, in this case, $\left(x_{1}, n_{1}, y_{1}\right)\left(y_{1}, n_{2}, y_{2}\right)=\left(x_{1}, n_{1}+n_{2}, y_{2}\right)$. The inverse of $(x, n, y)$ is $(y,-n, x)$. A basis of topology for $G$ is given by the sets

$$
Z(U, V, k, l)=\{(x, k-l, y) \in G: x \in U, y \in V\},
$$

where $U$ and $V$ are open subsets of $X$ such that $\left.T^{k}\right|_{U}$ and $\left.T^{l}\right|_{V}$ are homeomorphisms onto their images and $T^{k}(U)=T^{l}(V)$. The groupoid $G$ is étale so it admits a Haar system consisting of counting measures.

Let $A$ be a $C^{*}$-algebra, let $\alpha_{t}: \mathbb{R} \rightarrow \operatorname{Aut}(A)$ be a strongly continuous action, and let $\beta \in \mathbb{R}$. Recall ([1, Section 5.3]) that a state $\varphi$ on $A$ is said to be an $(\alpha, \beta)$-KMS state if

$$
\varphi\left(b \alpha_{i \beta}(a)\right)=\varphi(a b)
$$

for all $a, b$ entire for $\alpha$. If $\beta=0$, then $\varphi$ is an $\alpha$-invariant tracial state. The parameter $\beta$ is called the inverse temperature.

Let $G$ be a locally compact étale groupoid and let $c$ be a real-valued continuous cocycle. Then $c$ defines a one-parameter automorphism group $\alpha^{c}$ of $C^{*}(G)$ via

$$
\alpha_{t}^{c}(f)(\gamma)=e^{i t c(\gamma)} f(\gamma),
$$

for all $t \in \mathbb{R}, \gamma \in G$, and $f \in C_{c}(G)$ ([16, Section II.5]). Each probability measure $\mu$ on $G^{(0)}$ defines a state $\omega_{\mu}$ on $C^{*}(G)$ via

$$
\omega_{\mu}(f)=\int_{G^{(0)}} E(f) d \mu,
$$

for all $f \in C^{*}(G)$, where $E$ is the canonical expectation onto $C_{0}\left(G^{(0)}\right)$. A probability measure $\mu$ on $G^{(0)}$ is quasi-invariant under $G$ with Radon-Nikodým derivative $d r^{*} \mu / d s^{*} s=e^{-\beta c}$ if and only if the state $\omega_{\mu}$ of $C^{*}(G)$ is an $\left(\alpha^{c}, \beta\right)$-KMS state ([16, Proposition II.5.4]). Moreover, if $c^{-1}(0)$ is principal then every $\left(\alpha^{c}, \beta\right)$-KMS state of $C^{*}(G)$ is of the form $\omega_{\mu}$ for some quasi-invariant probability measure $\mu$ on $G^{(0)}$ with Radon-Nikodým derivative $d r^{*} \mu / d s^{*} \mu=e^{-\beta c}$ ([13, Proposition 3.2]). 
If $G$ is the Renault-Deaconu groupoid associated with a local homeomorphism $T$ on a compact metric space $(X, \rho)$, then every real valued continuous function $\varphi$ on $X$ defines a continuous one-cocycle on $G$ via the formula

$$
c_{\varphi}(x, m-n, y)=\sum_{i=0}^{m-1} \varphi\left(T^{i} x\right)-\sum_{i=0}^{n-1} \varphi\left(T^{i} y\right) .
$$

Moreover, every continuous one-cocycle on $G$ is of this form (see [3, Lemma 2.1]). In this case, the condition that $c_{\varphi}^{-1}(0)$ is principal means that ([13, page 2073])

$$
T^{n}(x)=x \text { and } n \geq 1 \Longrightarrow \varphi(x)+\varphi(T x)+\cdots+\varphi\left(T^{n-1} x\right) \neq 0 .
$$

This condition is trivially satisfied when $\varphi$ is strictly positive or strictly negative.

\section{The Main Result}

In this section we show that if $(X, \rho)$ is a compact metric space and $T: X \rightarrow X$ is a local homeomorphism which satisfies additional conditions as described below, then the Hausdorff measure gives rise to a KMS-state with inverse temperature the Hausdorff dimension. We will also show that the KMS state is unique under some mild hypotheses.

Definition 3.1. Let $(X, \rho)$ be a compact metric space and let $T: X \rightarrow X$ be a local homeomorphism. We say that $T$ satisfies the local scaling condition if

$$
(x, y) \mapsto \frac{\rho(T x, T y)}{\rho(x, y)}
$$

extends to a continuous function $f$ on $X \times X$ that is strictly positive on the diagonal $\Delta_{X}=\{(x, x) \mid x \in X\}$.

The next proposition provides an equivalent formulation of the local scaling property.

Proposition 3.2. Let $(X, \rho)$ be a compact metric space and let $T: X \rightarrow X$ be a local homeomorphism. Then $T$ satisfies the local scaling condition if and only if there is a continuous function $\varphi \in C(X, \mathbb{R})$ such that:

i. for all $x \in X$,

$$
e^{\varphi(x)}=\lim _{y \rightarrow x} \frac{\rho(T x, T y)}{\rho(x, y)} ;
$$

ii. for any $\varepsilon>0$ there is $\delta>0$ such that if $0<\rho(x, y)<\delta$, then

$$
\left|\frac{\rho(x, y)}{\rho(T x, T y)}-e^{-\varphi(x)}\right|<\varepsilon .
$$

Remark 3.3. Notice that since $T$ is assumed to be a local homeomorphism and $X$ is compact, there is $\delta_{1}>0$ such that if $0<\rho(x, y)<\delta_{1}$ then $T x \neq T y$. In the following we will implicitly assume that $\delta$ above satisfies $\delta \leq \delta_{1}$. This will ensure that inequality 3.1 makes sense.

Proof. Suppose that $f$ satisfies the local scaling condition. Then the function $\varphi$ : $X \rightarrow \mathbb{R}$, given by $\varphi(x)=\log f(x, x)$ for $x \in X$, is continuous on $X$; since $f$ is uniformly continuous on $X \times X$, it follows easily that the conditions above are satisfied. 
Conversely, suppose that $T$ satisfies the above conditions. We then define $f$ : $X \times X \mapsto \mathbb{R}$ via

$$
f(x, y)= \begin{cases}\frac{\rho(T x, T y)}{\rho(x, y)} & \text { if } x \neq y \\ e^{\varphi(x)} & \text { if } x=y .\end{cases}
$$

Then $f(x, x)>0$ for all $x \in X$ and it suffices to show that $f$ is continuous. Since $f$ is clearly continuous at $(x, y)$ if $x \neq y$, we need only prove that $f$ is continuous on the diagonal.

We prove first that $f$ is bounded on $X \times X$. Assume, by contradiction, that for each $n \in \mathbb{N}$ there are $x_{n}$ and $y_{n}$ in $X$ such that $\left|f\left(x_{n}, y_{n}\right)\right|>n$. Then there is a subsequence $\left(x_{n_{k}}, y_{n_{k}}\right)$ that converges to $(x, y) \in X \times X$. If $x \neq y$ then it follows that $x_{n_{k}} \neq y_{n_{k}}$ eventually, and that $\lim _{k \rightarrow \infty} f\left(x_{n_{k}}, y_{n_{k}}\right)=f(x, y)$. But this contradicts the fact that the sequence $\left|f\left(x_{n_{k}}, y_{n_{k}}\right)\right|$ is unbounded. Assume now that $x=y$. Since $\varphi$ is continuous on $X$ and thus bounded, we may assume (by passing to a subsequence if necessary) that $x_{n_{k}} \neq y_{n_{k}}$ for all $k$. Given $\varepsilon>0$, choose $\delta>0$ such that (3.1) holds. Then there is an integer $N \geq 1$ such that for all $k \geq N$

$$
\begin{aligned}
& \rho\left(x, x_{n_{k}}\right)<\delta / 2, \quad \rho\left(x, y_{n_{k}}\right)<\delta / 2 \quad \text { and } \\
& \left|1 / f(x, x)-1 / f\left(x_{n_{k}}, x_{n_{k}}\right)\right|<\varepsilon
\end{aligned}
$$

(by the continuity of $e^{-\varphi}$ ). It follows that for $k \geq N \rho\left(x_{n_{k}}, y_{n_{k}}\right)<\delta$ and, since $e^{-\varphi(x)}=1 / f(x, x)$, we also have that

$$
\left|\frac{1}{f\left(x_{n_{k}}, y_{n_{k}}\right)}-\frac{1}{f\left(x_{n_{k}}, x_{n_{k}}\right)}\right|<\varepsilon
$$

Therefore

$$
\begin{aligned}
\left|\frac{1}{f\left(x_{n_{k}}, y_{n_{k}}\right)}-\frac{1}{f(x, x)}\right| & \leq\left|\frac{1}{f\left(x_{n_{k}}, y_{n_{k}}\right)}-\frac{1}{f\left(x_{n_{k}}, x_{n_{k}}\right)}\right|+\left|\frac{1}{f\left(x_{n_{k}}, x_{n_{k}}\right)}-\frac{1}{f(x, x)}\right| \\
& <2 \varepsilon .
\end{aligned}
$$

Since $\left|1 / f\left(x_{n}, y_{n}\right)\right|<1 / n$ it follows that $1 / f(x, x)=0$, which is a contradiction. Thus $f$ is bounded on $X \times X$; let $M>0$ be a bound of the function.

We return now to the continuity of $f$ for $x=y$. Let $x_{n} \rightarrow x$ and $y_{n} \rightarrow x$. If $x_{n}=y_{n}$ for all $n$ then $\lim _{n \rightarrow \infty} f\left(x_{n}, x_{n}\right)=f(x, x)$ because $\varphi$ is assumed to be continuous. So, by passing to a subsequence, if necessary, we may assume that $x_{n} \neq y_{n}$ for all $n$. Let $\varepsilon>0$ be given; choose $\delta>0$ such that (3.1) holds. There is an integer $N$ such that for all $n \geq N$

$$
\begin{aligned}
& \rho\left(x, x_{n}\right)<\delta / 2, \quad \rho\left(x, y_{n}\right)<\delta / 2 \quad \text { and } \\
& \left|1 / f(x, x)-1 / f\left(x_{n}, x_{n}\right)\right|<\varepsilon .
\end{aligned}
$$

Then, for such $n$,

$$
\begin{aligned}
\left|f\left(x_{n}, y_{n}\right)-f(x, x)\right| & =\left|f\left(x_{n}, y_{n}\right) f(x, x)\right|\left|\frac{1}{f(x, x)}-\frac{1}{f\left(x_{n}, y_{n}\right)}\right| \\
& \leq M^{2}\left(\left|\frac{1}{f(x, x)}-\frac{1}{f\left(x_{n}, x_{n}\right)}\right|+\left|\frac{1}{f\left(x_{n}, x_{n}\right)}-\frac{1}{f\left(x_{n}, y_{n}\right)}\right|\right) \\
& <2 M^{2} \varepsilon .
\end{aligned}
$$

Therefore, $f$ is continuous on $X \times X$ and $T$ satisfies the local scaling condition. 
Theorem 3.4. Let $(X, \rho)$ be a compact metric space and let $T: X \rightarrow X$ be a local homeomorphism that satisfies the local scaling condition. Let $\varphi \in C(X, \mathbb{R})$ be such that

$$
e^{\varphi(x)}=\lim _{y \rightarrow x} \frac{\rho(T x, T y)}{\rho(x, y)},
$$

and let $c_{\varphi}$ be the associated one-cocycle on the Renault-Deaconu groupoid $G$ associated with $(X, T)$. Then the state $\omega_{\mu}$ given by the equation (2.1) is an $(\alpha, \beta)-K M S$ state where $\alpha=\alpha^{c_{\varphi}}, \beta=\operatorname{dim} X$, and $\mu=\mu^{\beta} / \mu^{\beta}(X)$.

Proof. As noted above in Section 2, by [16, Proposition II.5.4], it suffices to show that $\mu$ is a quasi-invariant measure on $G^{(0)}$ and

$$
\frac{d r^{*} \mu}{d s^{*} \mu}=e^{-\beta c_{\varphi}} \text {. }
$$

This is equivalent with

$$
\mu(U)=\int_{T U} e^{-\beta \varphi\left(\left(\left.T\right|_{U}\right)^{-1} x\right)} d \mu(x)
$$

for all open sets $U$ such that $\left.T\right|_{U}$ is a homeomorphism onto $T U$ ([19, Page 6]; see also [18]). We will call such sets sections of $T$.

By the local scaling property (see Definition 3.1) $(x, y) \mapsto \frac{\rho(T x, T y)}{\rho(x, y)}$ extends to a continuous function $f$ on $X \times X$ that is strictly positive on the diagonal. We know that $\varphi(x)=\log f(x, x)$ is uniformly continuous, since $f$ is continuous and $X$ is compact. This fact together with (3.1) implies that for any $\varepsilon>0$ there is $\delta>0$ such that if $0<\rho(x, y)<\delta$ then

$$
\left|\frac{\rho(x, y)}{\rho(T x, T y)}-e^{-\varphi(x)}\right|<\varepsilon
$$

and

$$
\left|e^{-\varphi(x)}-e^{-\varphi(y)}\right|<\varepsilon .
$$

Fix $\varepsilon>0$ such that $2 \varepsilon<\min _{x \in X} e^{-\varphi(x)}$. Suppose, first, that $U$ is a section of $T$ such that $\operatorname{diam} U<\delta$, where $\delta$ is chosen such that (3.4) and (3.5) hold. Let $\underline{x}, \bar{x} \in \bar{U}$ such that $e^{-\varphi(\underline{x})} \leq e^{-\varphi(x)} \leq e^{-\varphi(\bar{x})}$ for all $x \in U$, where $\bar{U}$ is the closure of the set $U$. Let $\varepsilon_{1}>0$ and suppose that $\mathcal{A}$ is an $\varepsilon_{1}$-cover of $U$ consisting of subsets of $U$ (see [5, page 167]). Then, for any $A \in \mathcal{A}$ we have that

$$
\operatorname{diam} A=\sup _{x, y \in A, x \neq y} \rho(x, y)=\sup _{x, y \in A, x \neq y} \rho(T x, T y) \frac{\rho(x, y)}{\rho(T x, T y)} .
$$

Now

and

$$
\left|\frac{\rho(x, y)}{\rho(T x, T y)}-e^{-\varphi(\underline{x})}\right|<2 \varepsilon
$$

$$
\left|\frac{\rho(x, y)}{\rho(T x, T y)}-e^{-\varphi(\bar{x})}\right|<2 \varepsilon
$$

for all $x, y \in A$ with $x \neq y$. Therefore

$$
\left(e^{-\varphi(\bar{x})}-2 \varepsilon\right) \operatorname{diam} T A \leq \operatorname{diam} A \leq\left(e^{-\varphi(\underline{x})}+2 \varepsilon\right) \operatorname{diam} T A .
$$

Hence

$$
\left(e^{-\varphi(\bar{x})}-2 \varepsilon\right)^{\beta} \bar{\mu}_{\varepsilon_{1}}^{\beta}(T U) \leq \bar{\mu}_{\varepsilon_{1}}^{\beta}(U) \leq\left(e^{-\varphi(\underline{x})}+2 \varepsilon\right)^{\beta} \bar{\mu}_{\varepsilon_{1}}^{\beta}(T U)
$$


and, by taking the limit when $\varepsilon_{1}$ goes to 0 ,

$$
\left(e^{-\varphi(\bar{x})}-2 \varepsilon\right)^{\beta} \mu(T U) \leq \mu(U) \leq\left(e^{-\varphi(\underline{x})}+2 \varepsilon\right)^{\beta} \mu(T U) .
$$

Let now $U$ be an arbitrary section of $T$. Then there is a finite disjoint family $\left\{U_{1}, \ldots, U_{N}\right\}$ of sections of $T$ such that $\bar{U}=\bigcup_{n=1}^{N} \bar{U}_{n}$ and $\operatorname{diam} U_{n}<\delta$ for $n=$ $1, \ldots, N$. Indeed, by the compactness of $\bar{U}$, there are finitely many open sets $\left\{V_{1}, \ldots, V_{N}\right\}$ such that $\bar{U} \subset \bigcup_{n=1}^{N} V_{n}$ and diam $V_{n}<\delta$ for $n=1, \ldots, N$. Now set $U_{1}=: U \cap V_{1}$ and

$$
U_{n+1}=: U \cap V_{n+1} \backslash\left(\bar{V}_{1} \cup \cdots \cup \bar{V}_{n}\right),
$$

for $n \geq 1$. It is routine to check that the $U_{n}$ satisfy the requisite conditions. Let $\underline{x}_{n}, \bar{x}_{n} \in \bar{U}_{n}$ such that $e^{-\varphi\left(\underline{x}_{n}\right)} \leq e^{-\varphi(x)} \leq e^{-\varphi\left(\bar{x}_{n}\right)}$ for all $x \in U_{n}$. Then, by inequality 3.6 ,

$$
\mu(U)=\sum_{n} \mu\left(U_{n}\right) \leq \sum_{n} \int_{T U_{n}}\left(e^{-\varphi\left(\underline{x}_{n}\right)}+2 \varepsilon\right)^{\beta} d \mu(x) .
$$

For all $x \in T U_{n}$ we have that $e^{-\varphi\left(\underline{x}_{n}\right)} \leq e^{-\varphi\left(\left(\left.T\right|_{U}\right)^{-1}(x)\right)}$. Thus

$$
\mu(U) \leq \int_{T U}\left(e^{-\varphi\left(\left(\left.T\right|_{U}\right)^{-1}(x)\right)}+2 \varepsilon\right)^{\beta} d \mu(x) .
$$

Similarly, since $e^{-\varphi\left(\left(\left.T\right|_{U}\right)^{-1}(x)\right)} \leq e^{-\varphi\left(\bar{x}_{n}\right)}$ for all $x \in T U_{n}$, we have that

$$
\int_{T U}\left(e^{\left.-\varphi\left(\left.T\right|_{U}\right)^{-1}(x)\right)}-2 \varepsilon\right)^{\beta} d \mu(x) \leq \mu(U) .
$$

Since $\varepsilon$ was arbitrarily chosen it follows that $(3.3)$ holds and the conclusion follows.

To ensure the uniqueness of the $(\alpha, \beta)$-KMS state we need to impose some conditions on the local homeomorphism $T$ and the map $\varphi$. We say that $T$ is positively expansive if there is an $\varepsilon>0$ such that for all $x \neq y$ there is an $n \in \mathbb{N}$ with $\rho\left(T^{n} x, T^{n} y\right) \geq \varepsilon$. We say that $T$ is exact if for every non-empty open set $U \subset X$ there is an $n>0$ such that $T^{n}(U)=X$. We say that a real-valued continuous function $\varphi$ on $X$ satisfies the Bowen condition with respect to $T$ (see [13, Definition $2.7])$ if there are $\delta, C>0$ such that

$$
\sum_{i=0}^{n-1} \varphi\left(T^{i} x\right)-\varphi\left(T^{i} y\right) \leq C,
$$

for all $x, y \in X$ and $n>0$ such that $\rho\left(T^{i} x, T^{i} y\right) \leq \delta$ for $0 \leq i \leq n-1$. Note that if $T$ is positively expansive and $\varphi$ is Hölder, that is, $|\varphi(x)-\varphi(y)| \leq k \rho(x, y)^{l}$ for some positive constants $k$ and $l$, then $\varphi$ satisfies the Bowen condition (see the discussion following [13, Definition 2.7]).

Proposition 3.5. Assume that the local homeomorphism $T: X \rightarrow X$ is positively expansive and exact, and assume that $\varphi$ satisfies the Bowen condition. Let $\alpha$ be the action on $C^{*}(G)$ determined by $c_{\varphi}$ and let $\beta$ be the Hausdorff dimension of $X$. If $T$ satisfies the local scaling condition and $c_{\varphi}^{-1}(0)$ is principal then $\beta$ is the unique inverse temperature which admits a KMS state for $\alpha$. Moreover, the $(\alpha, \beta)-K M S$ state $\omega_{\mu}$ is unique. 
Proof. Theorem 3.4 implies that there is a $(\alpha, \beta)$-KMS state, namely $\omega_{\mu}$. By Walters' version of the Ruelle-Perron-Frobenius Theorem ([13, Theorem 2.8], see also [19, Theorem 6.1], [24, Theorem 8], [26, Theorem 2.16]) there are unique $\lambda>0$ and probability measure $\nu$ such that

$$
\mathcal{L}_{-\varphi}^{*}(\nu)=\lambda \nu,
$$

where $\mathcal{L}_{-\varphi}(f)(x)=\sum_{T y=x} e^{-\varphi(y)} f(y)$ is the transfer operator associated with $\varphi$. Proposition 4.2 of [18] (see also [20, Proposition 3.4.1]) implies that $\beta$ is the unique inverse temperature which admits a KMS state for $\alpha$ and $\nu=\mu$.

Theorem 3.4 and [13, Theorem 3.5 i $)]$ imply that $P(T,-\beta \varphi)=0$, where $P(T, \cdot)$ is the topological pressure([25, Section 9.1]). Since $c_{\varphi}^{-1}(0)$ is principal, [13, Theorem $3.5 \mathrm{ii})$ ] implies that the $(\alpha, \beta)$-KMS state $\omega_{\mu}$ is unique.

In the last result of this section we show how one can compute the topological entropy of the local endomorphism $T$ under some suitable hypothesis. Recall that the topological entropy of $T, h(T)$, equals $P(T, 0)$.

Proposition 3.6. Let $T: X \rightarrow X$ be a local homeomorphism. Suppose that $T$ is positively expansive, exact, and satisfies the local scaling condition. Assume that the map $\varphi$ is constant, that is, there is a constant $\tau>1$ such that

$$
\tau=\lim _{y \rightarrow x} \frac{\rho(T x, T y)}{\rho(x, y)},
$$

for all $x \in X$. Then $h(T)=\beta \ln \tau$, where $\beta$ is the Hausdorff dimension of $X$.

Proof. By Theorem 3.4 and Proposition 3.5 there is a unique $(\alpha, \beta)$-KMS state $\omega_{\mu}$. Moreover, Theorem 3.5 of 13 implies that $P(T,-\beta \ln \tau)=0$. Since $P(T,-\beta \ln \tau)=$ $h(T)-\beta \ln \tau$, it follows that $h(T)=\beta \ln \tau$.

\section{EXAMPLES}

4.1. Cuntz algebras. Fix $n \in \mathbb{N}$ with $n>1$ and let $E=\{1, \ldots, n\}$ be the alphabet with $n$ letters. Let $\left(r_{1}, r_{2}, \ldots, r_{n}\right)$ be a list of positive numbers such that $r_{i}<1$ for all $i \in\{1, \ldots, n\}$. We call such a list a contractive ratio list (see [5, Chapter 4]). We set $X$ to be the infinite path space over the alphabet $E$, that is

$$
X=E^{\infty}=\left\{\left(x_{k}\right)_{k \in \mathbb{N}}: x_{k} \in E\right\} .
$$

We write $E^{k}$ for the set of paths of length $k$ over $E$ and we set $E^{*}=\bigcup_{k} E^{k}$. We define a metric on $X$ based on the given ratio list. For $\sigma \in E^{k}$ the cylinder of $\sigma$ is

$$
Z(\sigma)=\left\{\left(x_{m}\right) \in X: x_{0}=\sigma_{0}, \ldots, x_{k-1}=\sigma_{k-1}\right\} .
$$

We specify the metric on $X$ by requiring that the diameter of a cylinder $Z(\sigma)$, with $\sigma \in E^{k}$ for some k, to equal $r_{\sigma}:=r_{\sigma_{0}} r_{\sigma_{1}} \ldots r_{\sigma_{k-1}}$ (see [5, Section 4.2]). Thus for $x \neq y$

$$
\rho(x, y)=\left\{\begin{array}{ll}
\inf \left\{r_{\sigma}: x, y \in Z(\sigma)\right\} & \text { if } x_{0}=y_{0} \\
1 & \text { if } x_{0} \neq y_{0}
\end{array} .\right.
$$

Then the left shift, $T: X \rightarrow X, T\left(\left(x_{k}\right)\right)=\left(x_{k+1}\right)$, is a local homeomorphism on $X$. If $G$ is the corresponding Renault-Deaconu groupoid then $C^{*}(G)$ is isomorphic to the Cuntz algebra $\mathcal{O}_{n}$ ([16, Section III.2], 4]). Recall that the Cuntz algebra is 
the universal $C^{*}$-algebra generated by $n$ isometries $\left\{S_{i}\right\}_{i=1}^{n}$ satisfying the following relations

$$
S_{i}^{*} S_{j}=\delta_{i j} I \text { and } \sum_{i=1}^{n} S_{i} S_{i}^{*}=I .
$$

Under our identification the generating isometries of $\mathcal{O}_{n}$ are given by $S_{j}=1_{\Gamma_{j}}$, where $\Gamma_{j}=\{(j x, 1, x): x \in X\}$, for $j=1, \ldots, n$. A routine computation shows that $T$ satisfies the local scaling property and

$$
\lim _{y \rightarrow x} \frac{\rho(T x, T y)}{\rho(x, y)}=\frac{1}{r_{x_{0}}},
$$

that is, $\varphi(x)=-\log r_{x_{0}}$. Thus the automorphism $\alpha$ on $\mathcal{O}_{n}$ is determined by

$$
\alpha_{t}\left(S_{j}\right)=e^{-i t \log r_{j}} S_{j}
$$

The Hausdorff dimension $\beta$ of $X$ is the unique number that satisfies the equation ([5, Theorem 6.4.3])

$$
\sum_{i=1}^{n} r_{i}^{s}=1
$$

The Hausdorff measure on $X$ is the unique Borel measure that satisfies $\mu^{\beta}(Z(\sigma))=$ $r_{\sigma}^{\beta}$ for all $\sigma \in E^{*}$. Theorem 3.4 and Proposition 3.5 imply that there exists a KMS-state for $\alpha$ at temperature $\beta$ if and only if $\beta$ satisfies (4.3). The KMS state is unique in this case. We recover, thus, Theorem 2.2 of [6].

If $r_{1}=r_{2}=\cdots=r_{n}=1 / e$, then the inverse temperature is $\log n$, which is the main result in 14. Moreover the topological entropy of $T$ is $\log n$. More generally, let $s>0$ be arbitrary and set $r_{1}=r_{2}=\cdots=r_{n}=n^{-1 / s}$. Then $\left(r_{1}, \ldots, r_{n}\right)$ is a contractive ratio list. The Hausdorff dimension of $X$ is $\beta=s$ and $\varphi(x)=\frac{\log n}{s}$. The topological entropy of $T$ is still $\log n$.

4.2. Generalized gauge actions on Cuntz algebras. We still assume that $E=$ $\{1, \ldots, n\}$ is a finite alphabet and we let $X=E^{\infty}$ endowed with the product topology. Then $X$ is a compact topological space. Let $T$ be the left shift, as in the previous example. Fix now a continuous function $f$ on $X$ such that $f(x)>1$ for all $x \in X$. We define next a metric $\rho_{f}$ on $X$ with the property

$$
\lim _{y \rightarrow x} \frac{\rho_{f}(T x, T y)}{\rho_{f}(x, y)}=f(x) .
$$

Let $\sigma \in E^{*}$ and let $Z(\sigma)$ be the cylinder of $\sigma$ as defined in 4.1). We will write $|\sigma|$ for the length of the finite word $\sigma$. Define

$$
w_{\sigma}:=\max _{z, w \in Z(\sigma)}\left(\prod_{i=0}^{|\sigma|-1} f\left(T^{i} z\right) \cdot \prod_{i=0}^{|\sigma|-1} f\left(T^{i} w\right)\right)^{-1 / 2}
$$

Let $\alpha, \beta \in E^{*}$ such that $|\alpha|<|\beta|$ and $\alpha_{i}=\beta_{i}$ for $i=0, \ldots,|\alpha|-1$; in this case, write $\alpha<\beta$ ([5]). Since $f(x)>1$ for all $x \in X$, it follows that if $\alpha<\beta$ then $w_{\beta}>w_{\alpha}$ and $\lim _{n \rightarrow \infty} w_{\sigma \mid n}=0$ for $\sigma \in E^{\infty}$. Proposition 2.6.5 of [5] implies that there is a metric $\rho_{f}$ on $X$ such that the diameter of $Z(\alpha)=w_{\alpha}$ for all $\alpha \in E^{*}$ and, if $x, y \in X$ such that $x_{i}=y_{i}$ for $i=0, \ldots, k-1$ and $x_{k} \neq y_{k}$ then $\rho_{f}(x, y)=w_{\sigma}$, 
MARIUS IONESCU AND ALEX KUMJIAN

where $\sigma \in E^{k}$ such that $\sigma_{i}=x_{i}, i=0, \ldots, k-1$. Thus if $x, y \in X$ and the length of their common longest prefix $\sigma$ is at least one, then

$$
\rho_{f}(x, y)=w_{\sigma}:=\max _{z, w \in Z(\sigma)}\left(\prod_{i=0}^{|\sigma|-1} f\left(T^{i} z\right) \cdot \prod_{i=0}^{|\sigma|-1} f\left(T^{i} w\right)\right)^{-1 / 2} .
$$

Note that the maximum in 4.6 is attained for some $\bar{z}$ and $\bar{w}$ in $Z(\sigma)$ since the function $f$ is continuous and the cylinder $Z(\sigma)$ is a closed subset of the compact space $X$. Also, if $m=\min _{x \in X}|f(x)|$ and $M=\max _{x \in X}|f(x)|$, then

$$
\frac{1}{M^{|\sigma|}} \leq \rho_{f}(x, y) \leq \frac{1}{m^{|\sigma|}},
$$

where $\sigma$ is the longest common prefix of $x$ and $y$, for all $x$ and $y$ in $X$. Thus, since $m>1$ by hypothesis, for $x$ and $y$ in $X$ we have that $\rho_{f}(x, y)<\varepsilon$ if and only if there is $N \geq 1$ and $\sigma \in E^{N}$ such that $x, y \in Z(\sigma)$. Thus the metric $\rho_{f}$ generates the topology on $X$. If $\left(r_{1}, \ldots, r_{n}\right)$ is a contractive ratio list and $f(x)=1 / r_{x_{0}}$ we recover the metric from the previous example.

We prove next that $T$ satisfies the local scaling condition (see Definition 3.1) with respect to the metric $\rho_{f}$ with $\varphi=\log f$. First we need a lemma.

Lemma 4.1. Let $x, y \in X$ such that their longest common prefix $\sigma$ has length at least 2 . Then there are points $z_{i}, w_{i} \in Z(\sigma), i=1,2$ such that

$$
\left(f\left(z_{1}\right) f\left(w_{1}\right)\right)^{\frac{1}{2}} \leq \frac{\rho_{f}(T x, T y)}{\rho_{f}(x, y)} \leq\left(f\left(z_{2}\right) f\left(w_{2}\right)\right)^{\frac{1}{2}} .
$$

Proof. Observe that $w_{\sigma}=\rho_{f}(x, y)$ and $w_{\sigma^{\prime}}=\rho_{f}(T x, T y)$ (Equation 4.6p), where $\sigma^{\prime}:=\sigma_{1} \ldots \sigma_{|\sigma|-1}$. Then

$$
\frac{\rho_{f}(T x, T y)}{\rho_{f}(x, y)}=\frac{w_{\sigma^{\prime}}}{w_{\sigma}} .
$$

Note that if $z \in Z(\sigma)$ then $T z \in Z\left(\sigma^{\prime}\right)$. Conversely, if $z^{\prime} \in Z\left(\sigma^{\prime}\right)$ then $\sigma_{0} z^{\prime} \in Z(\sigma)$. Let $z_{1}, w_{1} \in Z(\sigma)$ the points for which the maximum in 4.6 is attained. That is,

$$
w_{\sigma}=\left(\prod_{i=0}^{|\sigma|-1} f\left(T^{i} z_{1}\right) \cdot \prod_{i=0}^{|\sigma|-1} f\left(T^{i} w_{1}\right)\right)^{-1 / 2} .
$$

Then $T z_{1}, T w_{1} \in Z\left(\sigma^{\prime}\right)$ and

$$
\begin{aligned}
w_{\sigma} & =\left(f\left(z_{1}\right) f\left(w_{1}\right)\right)^{-\frac{1}{2}} \cdot\left(\prod_{i=1}^{|\sigma|-1} f\left(T^{i} z_{1}\right) \cdot \prod_{i=1}^{|\sigma|-1} f\left(T^{i} w_{1}\right)\right)^{-1 / 2} \\
& \leq\left(f\left(z_{1}\right) f\left(w_{1}\right)\right)^{-\frac{1}{2}} \cdot w_{\sigma^{\prime}}
\end{aligned}
$$

Thus the first part of 4.7] holds. Let now $\bar{z}_{2}, \bar{w}_{2} \in Z\left(\sigma^{\prime}\right)$ such that

$$
w_{\sigma^{\prime}}=\left(\prod_{i=0}^{|\sigma|-2} f\left(T^{i} \bar{z}_{2}\right) \cdot \prod_{i=0}^{|\sigma|-2} f\left(T^{i} \bar{w}_{2}\right)\right)^{-1 / 2} .
$$


Then $z_{2}=\sigma_{0} \bar{z}_{2}$ and $w_{2}=\sigma_{0} \bar{w}_{2}$ belong to $Z(\sigma)$ and

$$
\left(\prod_{i=0}^{|\sigma|-1} f\left(T^{i} z_{2}\right) \cdot \prod_{i=0}^{|\sigma|-1} f\left(T^{i} w_{2}\right)\right)^{-1 / 2} \leq w_{\sigma}
$$

Thus

$$
\left(f\left(z_{2}\right) f\left(w_{2}\right)\right)^{-\frac{1}{2}} w_{\sigma^{\prime}} \leq w_{\sigma},
$$

which is the second part of 4.7 ).

Let $\varepsilon>0$ be given such that $\varepsilon<\min f(x)$. Since $f$ is uniformly continuous on $X$ there is a $\delta>0$ such that if $\sigma \in E^{*}$ is chosen so that $1 / m^{|\sigma|}<\delta$ then $|f(z)-f(w)|<\varepsilon$ for all $z, w \in Z(\sigma)$. Let $x, y \in X$ be such that the length of their common prefix $\sigma$ satisfies $1 / m^{|\sigma|}<\delta$. Suppose that $z_{1}, w_{1}$ and $z_{2}, w_{2}$ are the points in $Z(\sigma)$ for which the inequality (4.7) holds. Since $\left|f\left(z_{2}\right)-f(x)\right|<\varepsilon$ it follows that $f\left(z_{2}\right)<f(x)+\varepsilon$. Similarly, $f\left(w_{2}\right)<f(x)+\varepsilon, f(x)-\varepsilon<f\left(w_{1}\right)$ and $f(x)-\varepsilon<f\left(z_{1}\right)$. Since all the above quantities are positive, inequality (4.7) implies that

$$
f(x)-\varepsilon \leq \frac{\rho_{f}(T x, T y)}{\rho_{f}(x, y)} \leq f(x)+\varepsilon
$$

Therefore (4.4) holds and (3.1) also holds with $\varphi=\log f$; hence, $T$ satisfies the local scaling condition (by Proposition 3.2). Since the topology on the RenaultDeaconu groupoid $G$ associated to $(X, T)$ depends only on the topology on $X$ and not the underlying metric, if follows that it is still the case that $C^{*}(G) \simeq \mathcal{O}_{n}$. The associated automorphism $\alpha_{t}$ of $C^{*}(G)$ is given via

$$
\alpha_{t}(a)(x, m-k, y)=e^{i t c_{f}(x, m-k, y)} a(x, m-k, y),
$$

for all $(x, m-k, y) \in G$ and $a \in C_{c}(G)$, where the cocycle $c_{f}$ is given by

$$
c_{f}(x, m-k, y)=\log \left(\prod_{i=0}^{m-1} f\left(T^{i} x\right) / \prod_{i=0}^{k-1} f\left(T^{i} y\right)\right) .
$$

These actions generalize the gauge actions on $\mathcal{O}_{n}$ described in the previous example. To see this, recall from the previous example that, under our identification, $S_{j}=$ $1_{\Gamma_{j}}$, where $\Gamma_{j}=\{(j x, 1, x): x \in X\}$, for $j=1, \ldots, n$. If $f(x)=1 / r_{x_{0}}$ an easy computation shows that

$$
e^{i t c_{f}(x, m-k, y)} S_{j}(x, m-k, y)=e^{-i t \log r_{j}} S_{j}(x, m-k, y),
$$

and we recover (4.2). Assuming that the Hausdorff dimension $\beta$ of $X$ is strictly positive and finite, Theorem 3.4 implies that there exists a KMS-state $\omega_{\mu}$ on $\mathcal{O}_{n}$ for $\alpha$ at inverse temperature $\beta$.

If $\log f(x)$ satisfies the Bowen condition, then $\beta$ and $\mu$ are the unique solutions of the equation $\mathcal{L}_{f, \beta}^{*}(\mu)=\mu$, where

$$
\mathcal{L}_{f, \beta}(a)(x)=\sum_{j=1}^{n} f(j x)^{-\beta} a(j x),
$$

for all $x \in X$ and $a \in C(X)$ ([19, Proposition 7.1], [13, Theorem 2.8 and proof of Proposition 3.5]). In this case, $\omega_{\mu}$ is the unique KMS state for $\alpha$ on $\mathcal{O}_{n}$ by Proposition 3.5 . 
4.3. Graph $C^{*}$-algebras. Suppose that $E=\left(E^{0}, E^{1}, r, s\right)$ is a finite directed graph, where $r: E^{1} \rightarrow E^{0}$ and $s: E^{1} \rightarrow E^{0}$ are the range and source maps, respectively. Suppose that $\left\{r_{e}\right\}_{e \in E^{1}}$ is a list of positive numbers ${ }^{1}$ such that $r_{e}<1$ for all $e \in E^{1}$. We say that $\left\{r_{e}\right\}_{e \in E^{1}}$ is a contractive ratio list for the graph $E$ (see [5, Section 4.3]). A path of length $n$ in the graph $E$ is a finite sequence $\sigma=\sigma_{0} \sigma_{1} \ldots \sigma_{n-1}$ such that $\sigma_{i} \in E^{1}$ for all $i \in\{0, \ldots, n-1\}$ and $s\left(\sigma_{i}\right)=r\left(\sigma_{i+1}\right)$ for all $i \in\{0, \ldots, n-2\}$. Note that we adopt the recent convention from the theory of graph $C^{*}$-algebras when we define a path in a graph; namely, we switch the traditional role of $r$ and $s$ (see, for example, [15] for more details about this). We write $E^{n}$ for the set of paths of length $n$ and $E^{*}=\bigcup_{n} E^{n}$ for the set of finite paths in the graph $E$. We extend the definition of $r$ and $s$ to $E^{*}$ via $r(\sigma)=r\left(\sigma_{0}\right)$ and $s(\sigma)=s\left(\sigma_{n-1}\right)$ if $\sigma \in E^{n}$. The infinite path space $X$ is defined via

$$
X:=E^{\infty}:=\left\{\left(x_{n}\right)_{n \in \mathbb{N}}: x_{n} \in E^{1} \text { and } s\left(x_{n}\right)=r\left(x_{n+1}\right)\right\} .
$$

For an element $x \in E^{\infty}$ we set $r(x)=r\left(x_{0}\right)$. We say that the graph $E$ is irreducible (or strongly connected) if for every two elements $v, w \in E^{0}$ there is a path $\sigma \in E^{*}$ such that $r(\sigma)=v$ and $s(\sigma)=w$. For $v \in E^{0}$ we write $v E^{\infty}$ for the set of infinite sequences $x \in X$ such that $r(x)=v$. Similarly one can define $v E^{k}$ and $v E^{k} w$.

If $s$ is a positive real number, then $s$-dimensional Perron numbers for the graph $E$ are positive numbers $q_{v}$, one for each vertex $v \in E^{0}$, such that

$$
q_{v}^{s}=\sum_{w \in E^{0}, e \in v E^{1} w} r(e)^{s} q_{w}^{s}
$$

for all $v \in E^{0}$ (see, for example, [5, Section 6.6]). For an irreducible graph $E$ there is a unique number $s \geq 0$ such that Perron numbers exist ([5, Theorem 6.9.6]).

If $\sigma \in E^{*}$ then the cylinder $Z(\sigma)$ is

$$
Z(\sigma)=\left\{x \in E^{\infty}: x_{i}=\sigma_{i}, i=0, \ldots, n-1\right\} .
$$

Using [5, Proposition 2.6.5], one can show that there is a metric $\rho$ on $X$ such that the diameter of $Z(\sigma)$ equals $r_{\sigma} q_{s(\sigma)}$, for all $\sigma \in E^{*}$. It follows that the metric $\rho$ satisfies the property

$$
\rho(e x, e y)=r_{e} \rho(x, y) \text { for all } x, y \in s(e) E^{\infty} .
$$

Provided that the number $s$ which satisfies condition 4.9 is positive, the Hausdorff dimension $\beta$ of $X$ equals $s$ and the Hausdorff measure on $X$ is the unique Borel measure such that $\mu^{\beta}(Z(\sigma))=q_{s(\sigma)}^{\beta} r_{\sigma}^{\beta}$ for all $\sigma \in E^{*}$ ([5, Section 6.6]).

The left shift $T: X \rightarrow X$ defined by $T\left(x_{n}\right)=\left(x_{n+1}\right)$ is a local homeomorphism on $X$. We assume that the graph $E$ is irreducible and it satisfies condition (L) from [1] (or, equivalently since $E$ is finite, condition (I) from [2]). That is, we assume that every loop in $E$ has an exit. Under these assumptions if $G$ is the Renault-Deaconu groupoid associated to the pair $(X, T)$, then $C^{*}(G)$ is isomorphic to the graph $C^{*}$-algebra $C^{*}(E)([12,[11$; see also [15]). The local homeomorphism $T$ satisfies the local scaling property with

$$
\lim _{y \rightarrow x} \frac{\rho(T x, T y)}{\rho(x, y)}=\frac{1}{r_{x_{0}}},
$$

\footnotetext{
$1_{\text {the ratio }} r_{e}$ should not be confused with $r(e)$, the range of the edge $e$.
} 
that is, $\varphi(x)=-\log r_{x_{0}}$. The corresponding automorphism of $C^{*}(G)$ is defined then via

$$
\alpha_{t}\left(P_{v}\right)=P_{v} \text { for all } v \in E^{0}
$$

and

$$
\alpha_{t}\left(S_{e}\right)=e^{-i t \log r_{e}} S_{e}
$$

where $\left(\left\{P_{v}\right\}_{v \in E^{0}},\left\{S_{e}\right\}_{e \in E^{1}}\right\}$ is a universal Cuntz-Krieger family generating $C^{*}(G)$ $([11,15])$.

Theorem 3.4 and 3.5 imply that there exists a KMS-state for $\alpha$ at inverse temperature $\beta$ if and only if $\beta$ is the unique positive number for which Perron numbers exist. In this case, there is a unique $(\alpha, \beta)$-KMS state, namely

$$
\omega_{\mu}(f)=\int E(f) d \mu
$$

where $\mu$ is the normalized Hausdorff measure on $X$ and $E$ is the canonical expectation onto $C(X)$.

If $r_{e}=r \in(0,1)$ for all $e \in E^{1}$, then the Hausdorff dimension satisfies $\lambda=r^{-\beta}$, where $\lambda$ is the Perron-Frobenius eigenvalue of the vertex matrix $A_{E}$ of the graph $E$ (see, for example, [5, Theorem 6.9.6]). Proposition 3.6 implies that the topological entropy of $T$ is $\log \lambda$.

4.4. Generalized gauge actions on graph $C^{*}$-algebras. The construction of Example 4.2 extends to the graph $C^{*}$-algebras described in the previous example. Suppose that $X$ is the infinite path space of a finite directed graph $E=\left(E^{0}, E^{1}, r, s\right)$ which is irreducible and satisfies condition (L) of [13. We endow $X$ with the product topology so that it is a compact topological space and we let $T$ be the left shift map on $X$ as in the previous example. Fix a continuous function $f$ on $X$ such that $f(x)>1$ for all $x \in X$ and fix a list of positive number $\left\{q_{v}\right\}_{v \in E^{0}}$ such that

$$
f(x)>\frac{q_{s\left(x_{0}\right)}}{q_{r\left(x_{0}\right)}} \text { for all } x \in E^{\infty} .
$$

For example, if $q_{v}=1$ for all $v \in V$ then the above condition is trivially satisfied. We define the diameter of a cylinder $Z(\sigma), \sigma \in v E^{*}$, to be $w_{\sigma} \cdot q_{s(\sigma)}$, where

$$
w_{\sigma}:=\max _{z, w \in Z(\sigma)}\left(\prod_{i=0}^{|\sigma|-1} f\left(T^{i} z\right) \cdot \prod_{i=0}^{|\sigma|-1} f\left(T^{i} w\right)\right)^{-1 / 2}
$$

Since $f(x)>1$ and the numbers $q_{v}$ satisfy (4.10), it follows that if $\alpha, \beta \in E^{*}$ are such that $\alpha<\beta$ then $\operatorname{diam} Z(\alpha)<\operatorname{diam} Z(\beta)$ and $\lim _{n \rightarrow \infty} \operatorname{diam} Z\left(\left.\sigma\right|_{n}\right)=0$ for all $\sigma \in E^{\infty}$. Then [5, Proposition 2.6.5] implies that there is a metric $\rho_{f}$ on $v X$ such that the diameter of $Z(\sigma)$ with respect to $\rho_{f}$ equals $w_{\sigma} \cdot q_{s(\sigma)}$ for all $\sigma \in v E^{\infty}$, for each $v \in E^{0}$. Namely, if $x, y \in v E^{\infty}$ and $\sigma \in E^{*}$ is their longest common prefix, then

$$
\rho_{f}(x, y)=w_{\sigma} \cdot q_{s(\sigma)}
$$

Since $X$ is the finite disjoint union of $v X$ we can extend $\rho_{f}$ to a metric on $X$.

If $f(x)=1 / r_{x_{0}}$ for a contractive ratio list $\left\{r_{e}\right\}_{e \in E^{1}}$ and $\left\{q_{v}\right\}_{v \in E^{0}}$ are $s$-dimensional Perron numbers for the graph $E$ then the condition 4.10 is implied by (4.9). Thus we recover the metric from the previous example. 
One can easily see that if $x$ and $y$ have a common prefix $\sigma$ of length at least 2 then

$$
\frac{\rho(T x, T y)}{\rho(x, y)}=\frac{w_{\sigma^{\prime}}}{w_{\sigma}}
$$

because $s\left(\sigma^{\prime}\right)=s(\sigma)$, where $\sigma^{\prime}$ is obtained from $\sigma$ by removing the first entry $\left(\sigma^{\prime}=\sigma_{1} \ldots \sigma_{|\sigma|-1}\right)$. Then the analogue of Lemma 4.1 holds and $T$ satisfies the local scaling property (by Proposition 3.2 with respect to the metric $\rho_{f}$ with

$$
\lim _{y \rightarrow x} \frac{\rho(T x, T y)}{\rho(x, y)}=f(x),
$$

for all $x \in X$, and condition (3.1) holds with $\varphi=\log f$. Assuming that the graph $E$ is irreducible and satisfies condition [L] of [11, the $C^{*}$-algebra $C^{*}(G)$ of the Renault-Deaconu groupoid $G$ associated to $(X, T)$ is still isomorphic to the graph $C^{*}$-algebra $C^{*}(E)$. The associated action $\alpha_{t}$ on $C^{*}(G)$ is given via

$$
\alpha_{t}(a)(x, m-n, y)=e^{i t c_{f}(x, m-n, y)} a(x, m-n, y),
$$

for all $(x, m-n, y) \in G$ and $a \in C_{c}(G)$, where the cocycle $c_{f}$ is defined as in 4.8). These actions generalize the gauge actions described in the previous example. Indeed, a universal Cuntz-Krieger family on $C^{*}(E)$ is given by $\left(\left\{P_{v}\right\}_{v \in E^{0}},\left\{S_{e}\right\}_{e \in E^{1}}\right)$, where $P_{v}=1_{\Delta_{v}}$ and $S_{e}=1_{\Gamma_{e}}$, and

$$
\Delta_{v}=\left\{(x, 0, x) \in G^{0}: r(x)=v\right\}
$$

and

$$
\Gamma_{e}=\left\{(e x, 1, x) \in G: x \in s(e) E^{\infty}\right\} .
$$

Then an easy computation shows that

$$
\begin{aligned}
e^{i t c_{f}(x, m-n, y)} 1_{\Delta_{v}}(x, m-n, y) & =1_{\Delta_{v}}(x, m-n, y), \text { and } \\
e^{i t c_{f}(x, m-n, y)} 1_{\Gamma_{e}}(x, m-n, y) & =e^{i t \log r_{e}} 1_{\Gamma_{e}}(x, m-n, y) .
\end{aligned}
$$

Let $\beta$ be the Hausdorff dimension of $X$ with respect to the metric $\rho_{f}$ and assume that $0<\beta<\infty$. Theorem 3.4 implies that there exists a KMS state $\omega_{\mu}$ on the graph $C^{*}$-algebra $C^{*}(E)$ for $\alpha$ at inverse temperature $\beta$. Moreover, if $\log f$ satisfies the Bowen condition, then $\beta$ is the unique inverse temperature for which KMS states exist and $\omega_{\mu}$ is the unique $(\alpha, \beta)$-KMS state. The Hausdorff dimension $\beta$ and the measure $\mu$ are the unique solutions of the equation $\mathcal{L}_{f, \beta}^{*}(\mu)=\mu([19,13])$, where

$$
\mathcal{L}_{f, \beta}(a)(x)=\sum_{e \in E^{1}, s(e)=r(x)} f(e x)^{-\beta} a(e x) .
$$

We recover, thus, some of the results of [7].

4.5. Coverings of $\mathbb{T}$. Let $f:[0,1] \rightarrow \mathbb{R}$ be a continuous positive function such that $f(0)=f(1)$ and $n:=\int_{0}^{1} f(t) d t$ is a positive integer such that $n \geq 2$. For example, the constant function $n$ satisfies these requirements. Let $X=\mathbb{T}=\mathbb{R} / \mathbb{Z}$ and let $\rho$ the induced metric on $X$. We define $T: X \rightarrow X$ by

$$
T(x)=\int_{0}^{x} f(t) d t
$$

Then $T$ is a local homeomorphism. Moreover, $T$ is an $n$-fold covering map. One can easily compute that

$$
\lim _{y \rightarrow x} \frac{\rho(T x, T y)}{\rho(x, y)}=f(x)
$$


Thus $T$ satisfies the local scaling condition and $\varphi(x)=\log f(x)$. Since the Hausdorff dimension $\beta=1$, Theorem 3.4 implies that $\omega_{\mu}$ is an $(\alpha, 1)$-KMS state, where $\mu=\mu^{1}$. If $f$ is continuously differentiable and $f(x)>1$, then $\varphi=\log f$ is both positive and Hölder. Thus $c_{\varphi}^{-1}(0)$ is principal and $\varphi$ satisfies the Bowen condition. Moreover, $T$ is positively expansive and exact. Therefore, by Proposition 3.5. $\omega_{\mu}$ is the unique KMS state on $C^{*}(G)$.

Suppose now that $f(x)=n$ for all $x \in[0,1]$. Then $f$ is continuously differentiable and $f(x)>1$. Moreover, Proposition 3.6 implies that $h(T)=\log n$.

4.6. The Sierpinski octafold. Consider the triangle $Y$ with vertices $v_{1}=(1,0,0)$, $v_{2}=(0,1,0)$, and $v_{3}=(0,0,1)$ in $\mathbb{R}^{3}$. Let $f_{1}, f_{2}, f_{3}$ be the the restrictions to $Y$ of the linear maps defined by the matrices

$$
A_{1}=\left[\begin{array}{ccc}
1 & 1 / 2 & 1 / 2 \\
0 & 1 / 2 & 0 \\
0 & 0 & 1 / 2
\end{array}\right], A_{2}=\left[\begin{array}{ccc}
1 / 2 & 0 & 0 \\
1 / 2 & 1 & 1 / 2 \\
0 & 0 & 1 / 2
\end{array}\right], A_{3}=\left[\begin{array}{ccc}
1 / 2 & 0 & 0 \\
0 & 1 / 2 & 0 \\
1 / 2 & 1 / 2 & 1
\end{array}\right] .
$$

Note that for $i=1,2,3$, we have $f_{i}(Y) \subset Y$ and $f_{i}$ is a similarity with ratio $1 / 2$. So $\left(f_{1}, f_{2}, f_{3}\right)$ is an iterated function system $([9)$ and it admits a unique invariant set $K([9,5])$

$$
K=f_{1}(K) \bigcup f_{2}(K) \bigcup f_{3}(K) .
$$

The set $K$ is a copy of the Sierpinski gasket. Using the terminology from [10 and 23], we call the sets $f_{i}(K), i=1,2,3,1$-cells.

Now consider the octahedron in $\mathbb{R}^{3}$ with vertices at $( \pm 1,0,0),(0, \pm 1,0)$ and $(0,0, \pm 1)$; note that each face is isometric to $Y$ and $Y$ is one of the faces. We consider four copies of the Sierpinski gasket $K$ (one of which is $K$ itself) on alternating faces of the octahedron; so any two of the Sierpinski gaskets intersect only at a single point. Let $X$ be the union of the four Sierpinski gaskets. Note that each point in $X$ has a neighborhood which is similar to a neighborhood of the Sierpinski gasket. Thus $X$ is what is called in 22] a fractafold (see also 23] and 21]). We call $X$ the Sierpinski octafold. One can also think of the octafold as four copies of the Sierpinski gasket such that any two of them are glued at one of the vertices as in Figure 4.1, where the dotted curves mean that the two endpoints of the curve are identified.

The metric $\rho$ on $X$ is the restriction of the Euclidean metric to $X$. Therefore the Hausdorff dimension of $K$ is $\log 3 / \log 2$ (see, for example, [5, Theorem 6.5.4]). Since $X$ is the union of four copies of $K$ and the Hausdorff dimension of a union of sets equals the maximum of the Hausdorff dimension of each of the sets ( 5 , Theorem 6.1.7]) it follows that the Hausdorff dimension of $X$ is also $\log 3 / \log 2$. To describe the local homeomorphism, label the four copies of the Sierpinski gasket, which we will call 0 -cells, as 1,2,3, and 4. Let 11,12,13 be the three 1-cells of the 0-cell 1, and similarly for the other three 0-cells (see Figure 4.1). The local homeomorphism $T$ we define is uniquely determined by the following two properties

(1) The vertices of the 0 -cells are left fixed.

(2) The restriction to each 1-cell defines an affine homeomorphism onto the 0 -cell that shares exactly one vertex with the original 1-cell.

It is enough to indicate where the points that are the vertices of the 1-cells and are not vertices of the 0 -cells map. We call these points midpoints. Each midpoint may 
FiguRE 4.1. The Sierpinski octafold

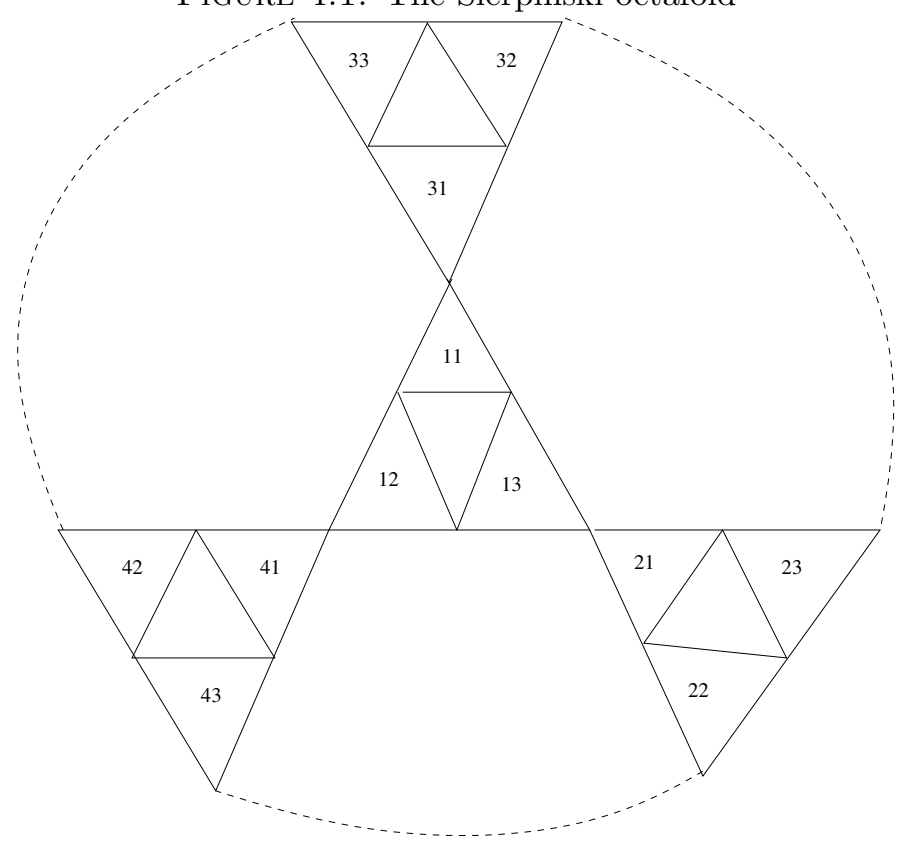

be written $.5\left(\epsilon_{1} e_{\sigma(1)}+\epsilon_{2} e_{\sigma(2)}\right)$ where $\epsilon_{i}= \pm 1, e_{i}$ is a canonical basis element of $\mathbb{R}^{3}$ and $\sigma$ is in the permutation group $S_{3}$. Then

$$
T\left(.5\left(\epsilon_{1} e_{\sigma(1)}+\epsilon_{2} e_{\sigma(2)}\right)\right)=-\epsilon_{1} \epsilon_{2} e_{\sigma(3)} .
$$

It is easy to see that this is well defined and $T$ is a local homeomorphism such that

$$
\lim _{y \rightarrow x} \frac{\rho(T x, T y)}{\rho(x, y)}=2 \text {. }
$$

It does not, however, satisfy condition (3.1) of Proposition 3.2 and, thus, it does not satisfy the local scaling condition. To see this, consider a point $y$ in the 1-cell 11 and another point $z$ in the 1-cell 12 such that they lie on the line segments, that form an angle of $\pi / 3$ and intersect at the midpoint $x$ (where the two 1-cells meet). We may also assume that they are equidistant from the midpoint $x$ (see Figure 4.1). Their images lie on adjacent edges of a square, so the Pythagorean Theorem implies that $\rho(T y, T z)=2 \sqrt{2} \rho(y, z)$, while $\rho(T x, T y)=2 \rho(x, y)$ and $\rho(T x, T z)=2 \rho(x, z)$. Since we can take $y$ and $z$ as close as we want to $x$, it follows that $T$ does not satisfy the local scaling property.

We claim, however, that the conclusion of Theorem 3.4 is still valid for this example with $\varphi=\log 2$. Since the proofs of Proposition 3.5 and Proposition 3.6 do not depend on the local scaling property and only on the existence of the KMS-state $\omega_{\mu}$, it follows that they are also valid, once we prove the claim.

Let $G$ be the associated Renault-Deaconu groupoid. The action on $C^{*}(G)$ is given via

$$
\alpha_{t}(f)(x, m-n, y)=e^{-i t(m-n) \log 2} f(x, m-n, y) .
$$

Recall from [16, Proposition II.5.4] that in order for the Hausdorff measure $\mu$ to be a quasi-invariant measure for $G$ the equality $(3.2)$ need only hold almost everywhere. 
One can modify the proof of Theorem 3.4 to show that this is the case for this example. Let $C$ be any of the 1-cells of the octafold. Then the restriction of $T$ to $C$ is a similarity with ratio 2 . Theorem 6.1 .9 of $[5]$ implies that $\mu(T U)=2^{\beta} \mu(U)$ for all subsets of $C$, where $\beta=\log 3 / \log 2$ is the Hausdorff dimension. In particular the equality is true for all open sections $U$ of $C$. It follows that the equation $(3.2)$ is true with $\varphi=\log 2$ for all points except possibly the midpoints and the conclusion of Theorem 3.4 is true for the octafold.

Thus, $\beta=\log 3 / \log 2$ is the unique inverse temperature which admits a KMS state on $C^{*}(G)$ for $\alpha$, the $(\alpha, \beta)$-KMS state $\omega_{\mu}$ given by (2.1) is unique, and the topological entropy is $h(T)=\log 3$.

\section{REFERENCES}

1. Ola Bratteli and Derek W. Robinson, Operator algebras and quantum statistical mechanics. 2, second ed., Texts and Monographs in Physics, Springer-Verlag, Berlin, 1997, Equilibrium states. Models in quantum statistical mechanics. MR MR1441540 (98e:82004)

2. Joachim Cuntz and Wolfgang Krieger, A class of $C^{*}$-algebras and topological Markov chains, Invent. Math. 56 (1980), no. 3, 251-268. MR MR561974 (82f:46073a)

3. V. Deaconu, A. Kumjian, and P. Muhly, Cohomology of topological graphs and Cuntz-Pimsner algebras, J. Operator Theory 46 (2001), no. 2, 251-264. MR MR1870406 (2003a:46093)

4. Valentin Deaconu, Groupoids associated with endomorphisms, Trans. Amer. Math. Soc. 347 (1995), no. 5, 1779-1786. MR MR1233967 (95h:46104)

5. Gerald Edgar, Measure, topology, and fractal geometry, second ed., Undergraduate Texts in Mathematics, Springer, New York, 2008. MR MR2356043 (2009e:28001)

6. David E. Evans, $O_{n} O_{n}$, Publ. Res. Inst. Math. Sci. 16 (1980), no. 3, 915-927. MR MR602475 (82g:46099)

7. Ruy Exel, KMS states for generalized gauge actions on Cuntz-Krieger algebras (an application of the Ruelle-Perron-Frobenius theorem), Bull. Braz. Math. Soc. (N.S.) 35 (2004), no. 1, 1-12. MR MR2057042 (2005c:46096)

8. R. Haag, N. M. Hugenholtz, and M. Winnink, On the equilibrium states in quantum statistical mechanics, Comm. Math. Phys. 5 (1967), 215-236. MR 0219283 (36 \#2366)

9. John E. Hutchinson, Fractals and self-similarity, Indiana Univ. Math. J. 30 (1981), no. 5, 713-747. MR MR625600 (82h:49026)

10. Jun Kigami, Analysis on fractals, Cambridge Tracts in Mathematics, vol. 143, Cambridge University Press, Cambridge, 2001. MR MR1840042 (2002c:28015)

11. Alex Kumjian, David Pask, and Iain Raeburn, Cuntz-Krieger algebras of directed graphs, Pacific J. Math. 184 (1998), no. 1, 161-174. MR MR1626528 (99i:46049)

12. Alex Kumjian, David Pask, Iain Raeburn, and Jean Renault, Graphs, groupoids, and CuntzKrieger algebras, J. Funct. Anal. 144 (1997), no. 2, 505-541. MR MR1432596 (98g:46083)

13. Alex Kumjian and Jean Renault, KMS states on $C^{*}$-algebras associated to expansive maps, Proc. Amer. Math. Soc. 134 (2006), no. 7, 2067-2078 (electronic). MR MR2215776 (2006m:46071)

14. Dorte Olesen and Gert K. Pedersen, Some $C^{*}$-dynamical systems with a single KMS state, Math. Scand. 42 (1978), no. 1, 111-118. MR MR500150 (80a:46041)

15. Iain Raeburn, Graph algebras, CBMS Regional Conference Series in Mathematics, vol. 103, Published for the Conference Board of the Mathematical Sciences, Washington, DC, 2005. MR MR2135030 (2005k:46141)

16. Jean Renault, A groupoid approach to $C^{*}$-algebras, Lecture Notes in Mathematics, vol. 793, Springer, Berlin, 1980. MR MR584266 (82h:46075)

17. _ Cuntz-like algebras, Operator theoretical methods (Timişoara, 1998), Theta Found., Bucharest, 2000, pp. 371-386. MR MR1770333 (2001g:46130)

18. _ AF equivalence relations and their cocycles, Operator algebras and mathematical physics (Constanța, 2001), Theta, Bucharest, 2003, pp. 365-377. MR MR2018241 (2005e:46129)

19. - The Radon-Nikodým problem for appoximately proper equivalence relations, Ergodic Theory Dynam. Systems 25 (2005), no. 5, 1643-1672. MR MR2173437 (2006h:46065) 
20. —,$C^{\star}$-algebras and dynamical systems, Publicações Matemáticas do IMPA. [IMPA Mathematical Publications], Instituto Nacional de Matemática Pura e Aplicada (IMPA), Rio de Janeiro, 2009, 27o Colóquio Brasileiro de Matemática. [27th Brazilian Mathematics Colloquium]. MR MR2536186

21. Robert S. Strichartz, Fractals in the large, Canad. J. Math. 50 (1998), no. 3, 638-657. MR MR1629847 (99f:28015)

22. __ Fractafolds based on the Sierpiński gasket and their spectra, Trans. Amer. Math. Soc. 355 (2003), no. 10, 4019-4043 (electronic). MR MR1990573 (2004b:28013)

23. _ Differential equations on fractals, Princeton University Press, Princeton, NJ, 2006, A tutorial. MR MR2246975 (2007f:35003)

24. Peter Walters, Invariant measures and equilibrium states for some mappings which expand distances, Trans. Amer. Math. Soc. 236 (1978), 121-153. MR MR0466493 (57 \#6371)

25. _ An introduction to ergodic theory, Graduate Texts in Mathematics, vol. 79, SpringerVerlag, New York, 1982. MR MR648108 (84e:28017)

26. _ Convergence of the Ruelle operator for a function satisfying Bowen's condition, Trans. Amer. Math. Soc. 353 (2001), no. 1, 327-347 (electronic). MR MR1783787 (2001g:37029)

Department of Mathematics, Colgate University, 13 Oak Dr, Hamilton, Ny 13346 , USA

E-mail address: mionescu@colgate.edu

Department of Mathematics, University of Nevada Reno NV 89557 USA

E-mail address: alex@unr.edu 